\title{
O PAPEL DA AVALIAÇÃO INICIAL SIMPLIFICADA NO PROGNÓSTICO DA PANCREATITE AGUDA
}

\author{
THE ROLE OF SIMPLIFIED INITIAL EVALUATION FOR ACUTE PANCREATITIS \\ PROGNOSIS
}

\author{
Márcio Cavalcante Carneiro,TCBC-RJ'1 ;osé Eduardo Ferreira Manso, TCBC-RJ²; \\ José Marcus Raso Eulálio, TCBC-RJ ${ }^{3}$; Juan Miguel Renteria, TCBC-RJ ${ }^{4}$; Mariana Dias Costa ${ }^{5}$
}

\begin{abstract}
RESUMO: Objetivo: Correlacionar a avaliação clínico-laboratorial inicial simplificada com a gravidade da pancreatite aguda e a presença de necrose. Método: Foi realizado um estudo retrospectivo dos pacientes com diagnóstico final de PA internados no Hospital Universitário Clementino Fraga Filho - UFRJ entre janeiro de 1990 e agosto de 2002. Foi considerado apenas o primeiro episódio de cada paciente. Os dados obtidos foram submetidos a análise estatística. Foram estudados 164 pacientes onde a idade média foi de 43,7 anos. Resultados: A etiologia biliar foi a mais freqüente com 43,9\% dos casos. A incidência de necrose foi de $21,3 \%$ e a mortalidade global de $23,2 \%$. Observamos que a ausência de taquicardia na admissão estava associada à forma branda da doença, e que os níveis plasmáticos de uréia e creatinina elevados na admissão estão associados à forma grave da doença, e a hiperglicemia $(>121 \mathrm{mg} / \mathrm{dl})$ à necrose pancreática. Conclusão: A avaliação inicial simplificada ainda tem espaço, embora que limitado, no acompanhamento do paciente com PA (Rev. Col. Bras. Cir. 2006; 33(3): 161-168).
\end{abstract}

Descritores: Pancreatite; Doença aguda; Pancreatite Necrosante Aguda; Prognóstico.

\section{INTRODUÇÃO}

Há aproximadamente um século tem sido dada grande atenção ao entendimento da fisiopatologia, do diagnóstico, da classificação e do tratamento da pancreatite aguda (PA). Obviamente durante este período grandes avanços foram conquistados, fundamentados em observações clínicas, estudos experimentais e a criação de novos recursos tecnológicos. Isto resultou na melhor compreensão desta doença com diminuição das taxas de mortalidade.

No entanto, muitas perguntas permanecem sem resposta, principalmente em relação à fisiopatologia visto que a etiologia da PA envolve mecanismos pouco conhecidos que convergem para um mecanismo central culminando com a ativação de enzimas pancreáticas, e iniciando um processo de autodigestão com conseqüente resposta inflamatória local e sistêmica. Também não é possível entender porquê um número de pacientes evolui com a forma grave da doença e outros não.

A dificuldade para entender e classificar esta doença é tanta que se reflete na variedade de terminologia destinada a classificar a PA. Vários simpósios internacionais foram realizados nas últimas quatro décadas visando definir e clas- sificar a PA. Um consenso foi estabelecido no simpósio de Atlanta realizado em Setembro de 1992, e há aproximadamente dez anos tem sido aceito como definição de pancreatite aguda branda e grave ${ }^{1}$.

Com otimismo pode-se aceitar que as mudanças em relação aos conceitos e classificações são frutos da aquisição de novas informações sobre esta doença. E mesmo havendo um consenso internacional, este é passível de questionamento, e não necessariamente atende a todas as questões pertinentes a PA. Portanto, se houver novas modificações deve-se tentar vê-las como resultado de uma melhor compreensão da doença.

A importância do reconhecimento da gravidade da PA foi enfatizado pelo Dr. Jonh Ranson em 1974 com a publicação do artigo "Objective Early Identification of Severe Acute Pancreatitis", publicado na revista "American Journal of Gastroenterology"2. Seu sistema de avaliação prognostica tornou-se mundialmente reconhecido, e a partir daí outros autores, como Imrie ${ }^{3}$,sugeriu modificações. Com isso, alguns serviços criaram seus próprios sistemas de classificação de gravidade na PA.

Diante de tantas tentativas em estabelecer o prognóstico da PA, pode-se perguntar: existe algum método eficaz

1. Cirurgião do Hospital Municipal Souza Aguiar; Professor Assistente da Faculdade de Medicina de Teresópolis; Mestre em Cirurgia Abdominal pela UFRJ- Programa de Pós-Graduação em Medicina (Cirurgia Geral).

2. Professor Adjunto do Departamento de Cirurgia da Faculdade de Medicina da UFRJ; Coordenador do Setor de Cirurgia do Pâncreas do HUCFFUFRJ; Cirurgião do Hospital Municipal da Piedade.

3. Professor Adjunto do Departamento de Cirurgia da Faculdade de Medicina da UFRJ; Cirurgião do Setor de Cirurgia do Pâncreas do HUCFF-UFRJ.

4. Cirurgião do Serviço de Emergência do Hospital Universitário Clementino Fraga Filho, UFRJ; Mestre e Doutor em Cirurgia Abdominal pela UFRJ

- do Programa de Pós-Graduação em Medicina (Cirurgia Geral) da UFRJ.

5. Aluna do Curso de Graduação em Medicina - UFRJ.

Recebido em 19-12-2005

Aceito para publicação em 23-01-2006

Conflito de interesses: nenhum

Fonte de financiamento: nenhuma

Trabalho realizado no Hospital Universitário Clementino Fraga Filho - UFRJ - Rio de Janeiro - RJ. 
em diagnosticar precocemente a possibilidade do paciente evoluir com a forma grave desta doença?

Este estudo retrospectivo tem como objetivo avaliar o perfil dos pacientes com PA atendidos neste hospital, assim como estudar o papel da avaliação clínico-laboratorial inicial simplificada e sua relação com a gravidade e a presença de necrose.

\section{MÉTODO}

Foi feito um estudo retrospectivo dos pacientes internados no Hospital Universitário Clementino Fraga Filho (HUCFF) com o diagnóstico de PA com alta ou óbito entre 1 de janeiro de 1990 e 9 de agosto de 2002.

À partir deste levantamento foram obtidos 293 prontuários que foram submetidos a estudo, tendo sido aproveitados 164 prontuários que atendiam aos critérios de inclusão.

Foram incluídos os casos de PA com quadro clínico compatível e dosagem de amilase sérica pelo menos três vezes o valor normal (valor de referência até $220 \mathrm{U} / \mathrm{L}$ ), casos de PA confirmados pela TC e ainda casos de achado operatório compatível com PA. Foi considerado apenas o primeiro episódio de cada paciente a fim de manter a independência dos casos.

Os critérios utilizados para definir PA grave são os estabelecidos no simpósio de Atlanta e incluem complicações locais (coleções ou necrose) e complicações sistêmicas (insuficiências orgânicas).

Os dados do exame físico selecionados para análise foram: dor abdominal do tipo difusa, peristalse diminuída ou ausente, defesa abdominal, peritonite, obstipação, febre (Temperatura axilar $\left.>37,4^{\circ} \mathrm{C}\right)$, taquicardia $(\mathrm{FC}>100 \mathrm{BPM})$, icterícia, taquipnéia (FR> 20 IRPM), hipotensão (PAS $<90 \mathrm{mmHg}$ ), hemorragia digestiva, oligúria.

Os dados de laboratório avaliados neste estudo foram: amilase (U/L), hematócrito (\%), leucograma (leucócitos/mm3), glicemia ( $\mathrm{mg} \%)$, uréia $(\mathrm{mg} \%)$, creatinina $(\mathrm{mg} \%)$, sódio $(\mathrm{mEq} / \mathrm{l})$, potássio $(\mathrm{mEq} / \mathrm{l})$. Foram selecionados estes dados pois são comumente solicitados na avaliação de pacientes durante o atendimento de emergência, por isso simples e acessíveis.Os dados de laboratório selecionados foram os da primeira medida nas primeiras 48 horas de internação.

Os pacientes foram divididos em três grupos: PAbranda, PA grave sem necrose e PA grave com necrose.

Com o objetivo de verificar a existência de diferença significativa nas variáveis estudadas entre dois grupos, foram aplicados os seguintes métodos: para comparação de proporções foi utilizado o Teste de Qui-quadrado $\left(\mathrm{k}^{2}\right)$ ou o Teste Exato de Fisher, quando o Teste de Qui-quadrado não pôde ser avaliado devido ao pequeno tamanho da amostra. A comparação de variáveis quantitativas entre os grupos foi analisada pelo teste de Análise de Variância de KruskalWallis, e complementada pelo Teste de Comparações Múltiplas quando havia diferença estatística significativa.

O critério de determinação de significância adotado foi o nível de $5 \%$, ou seja, quando o valor de $p$ do teste estatístico for menor ou igual a 0,05 .

\section{RESULTADOS}

Dos 164 pacientes, $88(53,7 \%)$ foram do sexo masculino e $76(46,3 \%)$ do sexo feminino.

As informações a respeito da idade, início dos sintomas até a admissão (tempo de evolução) e o tempo de internação estão resumidas na Tabela 1 .

Segundo os critérios de Atlanta, foram considerados como PA grave $74(45,1 \%)$ pacientes e como PA branda 90 $(54,9 \%)$.

Quanto a etiologia foi observado a seguinte proporção: alcoólica 41 (25\%), biliar 72 (43,9\%), outras 51 (31,1\%).

O tratamento operatório foi realizado em 87 (53\%) pacientes Neste grupo estão incluídas as cirurgias de urgência e as cirurgias eletivas (sendo estas últimas cirurgias realizadas na mesma internação após recuperação do episódio de PA como, por exemplo, a colecistectomia, as anastomoses biliodigestivas e o tratamento cirúrgico de pseudocisto).

Dos casos operados, os achados de complicações pancreáticas foram necrose em $30(34,5 \%)$ pacientes, abscesso em $18(20,7 \%)$, pseudocisto em $8(9,2 \%)$ e coleção sem cápsula em 4 (4,6\%).Os demais não apresentavam sinais destas complicações.

No grupo estudado de 164 pacientes, 77 pacientes não foram operados e 30 (18,3\%) estiveram internados no CTI.

A mortalidade global foi de $38(23,2 \%)$ pacientes. Sendo que, a mortalidade no grupo com PA branda foi de 3 $(3,3 \%)$ pacientes, e no grupo com PA grave, segundo os critérios de Atlanta, foi de 35 (47,3\%). No grupo de PA grave com necrose a mortalidade foi de $23(65,7)$ pacientes e no grupo de PA grave sem necrose a mortalidade foi de 12 (34,3\%).

A presença de necrose pancreática seja confirmada por TC, cirurgia ou ambos estava presente em 35 (21,3\%) dos pacientes. Considerando apenas os 74 pacientes graves, os 35 pacientes com necrose corresponderam a 47,3\%. Enquanto que, $39(52,7 \%)$ pacientes com PA grave apresentaram disfunção orgânica ou coleção pancreática sem necrose pancreática associada.

Tabela 1 - Dados sobre idade, tempo de evolução e internação.

\begin{tabular}{lcclccc}
\hline Variável & $\boldsymbol{n}$ & Média & D.P. & Mediana & Mínimo & Máximo \\
\hline Idade (anos) & 160 & 43,7 & 14,5 & 41,5 & 18 & 86 \\
Evolução (dias) & 145 & 7,1 & 11 & 3 & 1 & 90 \\
Internação (dias) & 148 & 22,9 & 20,8 & 16 & 1 & 170 \\
\hline
\end{tabular}

D.P.: Desvio Padrão. 
Tabela 2 - Frequência de achado de exame físico.

\begin{tabular}{llc}
\hline Sinal ou Sintoma & N & \% \\
\hline Dor abdominal difusa & 31 & 18,9 \\
Peristalse diminuída ou ausente & 42 & 25,6 \\
Defesa abdominal & 37 & 22,6 \\
Descompressão dolorosa & 38 & 23,2 \\
Obstipação & 08 & 4,9 \\
Febre & 33 & 20,1 \\
Taquicardia & 29 & 17,7 \\
Taquipnéia & 17 & 10,4 \\
Icterícia & 43 & 26,2 \\
Hipotensão & 07 & 4,3 \\
Hemorragia digestiva & 03 & 1,8 \\
\hline
\end{tabular}

Os sinais e sintomas que poderiam estar associados a gravidade da PA, e que foram pesquisados no exame físico inicial encontram-se na Tabela 2.

Dos 164 casos estudados a dosagem de exames laboratoriais nas primeiras 48 horas não estava presente em todos pacientes. Os exames, a freqüência e os resultados encontrados são mostrados na Tabela 3.

Dentre os pacientes estudados, 77 (47\%) foram submetidos ao exame de TC. Destes, 27 (35\%) foram realizados com administração de contraste venoso.

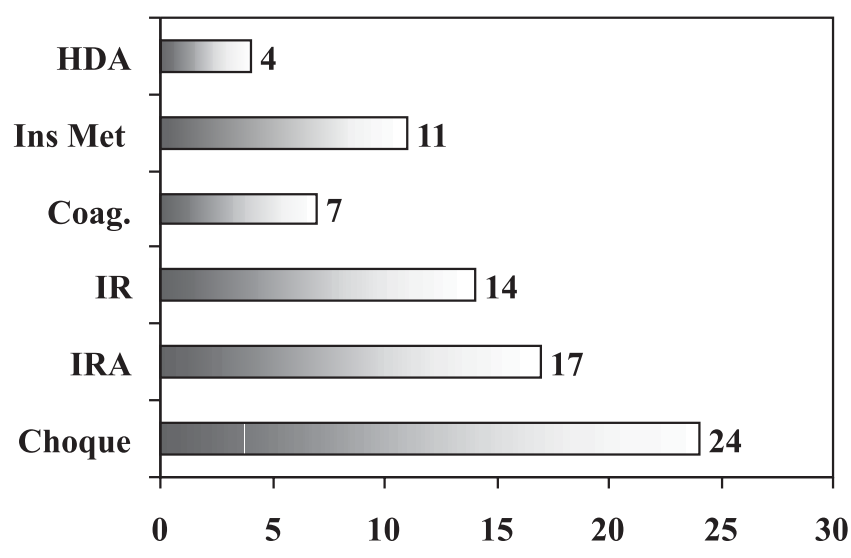

Figura 1 - Presença de disfunção orgânica.
Nos pacientes em que a TC foi realizada, os achados de complicações pancreáticas locais, utilizados para a classificação da gravidade, foram: coleção líquida retroperitonial em $20(26 \%)$ pacientes, necrose pancreática em seis $(7,8 \%)$ pacientes.

A presença de falência orgânica foi pesquisada separadamente para cada tipo de disfunção. E a sua distribuição encontra-se na Figura 1.

O estudo da relação entre a idade, tempo de evolução e tempo de internação entre os grupos estudados são mostrados na Tabela 4.

O estudo do tempo de internação evidenciou diferença significativa no grupo de PA grave com necrose com valor de $p$ igual a 0,001 que apresentou tempo médio de internação maior que o grupo com PA branda. Não houve diferença significativa entre os demais pares de grupos, isto é, entre PA grave com necrose e sem necrose e entre PA grave sem necrose e PA branda.

Aplicando o teste estatístico, verificou-se que a proporção de taquicardia no grupo com PA branda $(7,8 \%)$ foi significativamente menor que nos grupos com PA grave com necrose $(25,7 \%)$ e PA grave sem necrose $(33,3 \%)$, com $p$ igual a 0,007 e 0,0001 respectivamente

A análise de dados como obstipação, hemorragia digestiva e oligúria ficou comprometida devido ao pequeno número de casos. Os dados referentes ao exame físico e sua relação com a gravidade são mostrados na Tabela 5 .

Para calcular a sensibilidade e especificidade da presença de taquicardia em relação a gravidade, foi considerado grupo de PA branda versus o grupo de PA grave com e sem necrose (visto que não houve diferença entre estes). Encontramos sensibilidade de $29 \%$ e especificidade de $92 \%$.

A avaliação dos dados laboratoriais em relação aos três grupos foi realizada pela Análise de Variância de KruskalWallis e complementada pelo Teste de Comparações Múltiplas. Estes dados são mostrados na Tabela 6 .

Após realização de análise estatística foi observado que existe diferença significativa no nível médio da glicemia entre os grupos $(p=0,03)$. Pelo teste de comparações múltiplas, identificou-se que o grupo de pacientes com PA grave e necrose apresentou glicemia média significativamente maior que os grupos com PA branda e PA grave sem necrose. Não existiu diferença significativa nos níveis de glicemia entre os grupos com PA grave sem necrose e PA branda.

Tabela 3 - Exames Laboratoriais.

\begin{tabular}{|c|c|c|c|c|c|c|}
\hline Variável & $\mathbf{n}$ & Média & D.P. & Mediana & Mínimo & Máximo \\
\hline Amilase & 111 & 1534,05 & 1957,38 & 956 & 7,9 & 11332 \\
\hline Leuco & 128 & 13668,00 & 7350,74 & 11900 & 1360 & 44800 \\
\hline Glicemia & 128 & 156,63 & 112,77 & 116 & 51 & 708 \\
\hline Uréia & 112 & 49,77 & 45,29 & 35,5 & 4 & 342 \\
\hline Creatinina & 122 & 1,62 & 2,40 & 1 & 0,4 & 22 \\
\hline Sódio & 121 & 136,18 & 6,50 & 136 & 117 & 153 \\
\hline Potássio & 125 & 4,00 & 0,75 & 3,9 & 2,4 & 6,6 \\
\hline $\mathrm{Ht}$ & 128 & 39,71 & 8,31 & 40,65 & 13 & 56 \\
\hline
\end{tabular}

DP - Desvio padrão. 
Tabela 4 - Dados relativos a idade, tempo de evolução e internação entre os grupos.

\begin{tabular}{|c|c|c|c|c|c|c|c|c|}
\hline Variável & Pancreatite & $\mathbf{n}$ & Média & D.P. & Mediana & Mínimo & Máximo & $p$ valor \\
\hline \multirow[t]{3}{*}{ Idade (anos) } & $\mathrm{PA} \mathrm{c} /$ necrose & 34 & 47,4 & 15,17 & 45 & 19 & 86 & 0,18 \\
\hline & PA s/ necrose & 39 & 41,2 & 13,0 & 39 & 19 & 76 & \\
\hline & PA branda & 87 & 43,3 & 14,8 & 42 & 18 & 86 & \\
\hline \multirow[t]{3}{*}{ Evolução (dias) } & PA c/ necrose & 31 & 5,2 & 5,4 & 3 & 1 & 20 & 0,76 \\
\hline & PA s/ necrose & 37 & 8,5 & 15,4 & 4 & 1 & 90 & \\
\hline & PA branda & 77 & 7,1 & 10,1 & 4 & 1 & 60 & \\
\hline \multirow[t]{3}{*}{ Internação(dias) } & $\mathrm{PA} \mathrm{c} /$ necrose & 30 & 35,7 & 33,2 & 28 & 3 & 170 & 0,001 \\
\hline & PA s/ necrose & 34 & 24,0 & 15,7 & 22,5 & 1 & 52 & \\
\hline & PA branda & 84 & 17,9 & 14,1 & 15 & 2 & 90 & \\
\hline
\end{tabular}

Foi observado ainda que existe diferença significativa no nível médio de uréia sérica entre os grupos $(p=0,0005)$. Pelo teste de comparações múltiplas, identificou-se que o grupo de pacientes com PA branda apresentou nível de uréia sérica média significativamente menor que o grupo com PA grave com e sem necrose. Não existiu diferença significativa nos níveis de uréia sérica entre os grupos com PA grave com e sem necrose.

Verificou-se que existe diferença significativa no nível médio da creatinina sérica entre os grupos $(p=0,0001)$. Pelo teste de comparações múltiplas identificou-se que o grupo com PA branda apresentou creatinina sérica média significativamente menor que os grupos de PA grave com e sem necrose. Não existe diferença significativa nos níveis de creatinina entre os grupos de PA grave com e sem necrose.

Finalmente observou-se que existe diferença significativa no nível médio de potássio sérico entre os grupos $(p=$ $0,028)$. Pelo teste de comparações múltiplas, identificou-se que o grupo com PA grave sem necrose apresentou níveis de potássio sérico médio significativamente maior que o grupo com PA branda. Não existe diferença significativa no nível de potássio sérico entre os demais pares de grupos. No entanto os valores encontravam-se dentro da faixa de normalidade.
Não houve diferença significativa nas demais variáveis entre os três grupos estudados.

Após a determinação dos exames laboratoriais que apresentaram diferença entre os grupos, estes foram submetidos a análise para determinação do melhor ponto de corte. Foi excluída desta análise a dosagem de potássio, pois mesmo havendo diferença entre os grupos, os níveis séricos médios encontravam-se dentro da normalidade em todos os grupos.

Para a análise do ponto de corte da glicemia em relação a presença de PA grave com necrose, foi considerado como grupo controle os pacientes com PA branda e PA grave sem necrose. Foram excluídos trinta e seis pacientes devido a falta de informação. Nesta população, segundo a curva ROC (Receiver Operator Characteristic), observou-se que o melhor ponto de corte para a glicemia foi maior que $121 \mathrm{mg} / \mathrm{dl}$, com sensibilidade de $63,3 \%$ e especificidade de $61,2 \%$.

Para a análise do ponto de corte dos níveis séricos da uréia e creatinina em relação a gravidade foi considerado como grupo controle os pacientes com PA branda. O ponto de corte encontrado para dosagem de uréia sérica foi maior que $36 \mathrm{mg} / \mathrm{dl}$ (com sensibilidade de 68,6\% e especificidade de

Tabela 5 - Distribuição dos dados de exames físicos entre os grupos.

\begin{tabular}{|c|c|c|c|c|c|c|}
\hline \multirow{2}{*}{$\frac{\text { Sinal/Sintoma }}{\text { Dor abdominal }}$} & \multirow{2}{*}{$\frac{\text { Presença }}{\mathrm{P}}$} & \multicolumn{2}{|c|}{ PA Grave c/ necrose } & PA Grave s/necrose & \multirow{2}{*}{$\frac{\text { PA Branda }}{12(13,3 \%)}$} & \multirow{2}{*}{$\frac{\mathbf{p}}{0,13}$} \\
\hline & & 9 & $(25,7 \%)$ & $10 \quad(25,6 \%)$ & & \\
\hline Difusa & $\mathrm{N}$ & 26 & $(74,3 \%)$ & $29 \quad(74,4 \%)$ & $78(86,7 \%)$ & \\
\hline \multirow[t]{2}{*}{ Febre } & $\mathrm{P}$ & 7 & $(20,0 \%)$ & $8(20,5 \%)$ & $18(20,0 \%)$ & 0,99 \\
\hline & $\mathrm{N}$ & 28 & $(80,0 \%)$ & $31 \quad(79,5 \%)$ & $72(80,0 \%)$ & \\
\hline \multirow{2}{*}{ Defesa Abdominal } & $P$ & 12 & $(34,3 \%)$ & $9(23,1 \%)$ & $16(17,8 \%)$ & 0,14 \\
\hline & $\mathrm{N}$ & 23 & $(65,7 \%)$ & $30 \quad(76,9 \%)$ & $74(82,2 \%)$ & \\
\hline Descompressão & $P$ & 10 & $(28,6 \%)$ & $9(23,1 \%)$ & $19(21,1 \%)$ & 0,67 \\
\hline Dolorosa & $\mathrm{N}$ & 25 & $(71,4 \%)$ & $30 \quad(76,9 \%)$ & $71(78,9 \%)$ & \\
\hline \multirow[t]{2}{*}{ Taquicardia } & $\mathrm{P}$ & 9 & $(25,7 \%)$ & $13 \quad(33,3 \%)$ & $7 \quad(7,8 \%)$ & 0,001 \\
\hline & $\mathrm{N}$ & 26 & $(74,3 \%)$ & $26 \quad(66,7 \%)$ & $83(92,2 \%)$ & \\
\hline \multirow[t]{2}{*}{ Icterícia } & $\mathrm{P}$ & 7 & $(20,0 \%)$ & $10 \quad(25,6 \%)$ & $26(28,9 \%)$ & 0,59 \\
\hline & $\mathrm{N}$ & 28 & $(80,0 \%)$ & $29 \quad(74,4 \%)$ & $64(71,1 \%)$ & \\
\hline \multirow[t]{2}{*}{ Taquipnéia } & $\mathrm{P}$ & 6 & $(17,1 \%)$ & $5(12,8 \%)$ & $6 \quad(6,7 \%)$ & 0,17 \\
\hline & $\mathrm{N}$ & 29 & $(82,9 \%)$ & $34 \quad(87,2 \%)$ & $84(93,3 \%)$ & \\
\hline Peristalse Diminuída & $\mathrm{P}$ & 13 & $(37,1 \%)$ & $11 \quad(28,2 \%)$ & $18(20,0 \%)$ & 0,13 \\
\hline ou Ausente & $\mathrm{N}$ & 22 & $(62,9 \%)$ & $28 \quad(71,8 \%)$ & $72(80,0 \%)$ & \\
\hline
\end{tabular}


Tabela 6 - Dados laboratoriais distribuídos entre os grupos.

\begin{tabular}{|c|c|c|c|c|c|c|c|c|}
\hline Variável & Pancreatite & $\mathbf{n}$ & Média & D.P. & Mediana & Mínimo & Máximo & $p$ valor \\
\hline \multirow[t]{3}{*}{ Amilase } & $\mathrm{PA} \mathrm{c} /$ necrose & 25 & 1076,4 & 1122,8 & 954 & 16 & 3800 & 0,32 \\
\hline & PA s/ necrose & 19 & 1738,7 & 2586,2 & 777 & 116 & 11332 & \\
\hline & PA branda & 67 & 1647,0 & 1999,7 & 1082 & 7,9 & 10500 & \\
\hline \multirow[t]{3}{*}{ Leuco } & PA c/ necrose & 27 & 15096 & 8173 & 14600 & 5000 & 44800 & 0,50 \\
\hline & PA s/ necrose & 29 & 14290 & 8722 & 11200 & 2400 & 33200 & \\
\hline & PA branda & 72 & 12883 & 6374 & 11250 & 1360 & 34500 & \\
\hline \multirow[t]{3}{*}{ Glicemia } & PA c/ necrose & 30 & 210,5 & 160,0 & 140 & 51 & 708 & 0,030 \\
\hline & PA s/ necrose & 27 & 140,3 & 91,5 & 108 & 70 & 479 & \\
\hline & PA branda & 71 & 140,1 & 87,9 & 114 & 68 & 540 & \\
\hline \multirow[t]{3}{*}{ Uréia } & PA c/ necrose & 27 & 59,5 & 41,9 & 51 & 8 & 185 & 0,0005 \\
\hline & PA s/ necrose & 24 & 74,1 & 70,9 & 56,5 & 16 & 342 & \\
\hline & PA branda & 61 & 35,9 & 25,2 & 30 & 4 & 180 & \\
\hline \multirow[t]{3}{*}{ Creat } & PA c/ necrose & 28 & 1,9 & 1,4 & 1,5 & 0,5 & 6,4 & 0,0001 \\
\hline & PA s/ necrose & 27 & 2,3 & 4,1 & 1,2 & 0,6 & 22 & \\
\hline & PA branda & 67 & 1,2 & 1,7 & 1 & 0,4 & 15 & \\
\hline \multirow[t]{3}{*}{ Sódio } & $\mathrm{PA} \mathrm{c} /$ necrose & 28 & 135,4 & 7,5 & 137 & 117 & 147 & 0,67 \\
\hline & PA s/ necrose & 29 & 135,5 & 6,5 & 136 & 122 & 148 & \\
\hline & PA branda & 64 & 136,8 & 6,1 & 137 & 125 & 153 & \\
\hline \multirow[t]{3}{*}{ Potássio } & PA c/ necrose & 29 & 4,1 & 0,8 & 4 & 2,7 & 5,8 & 0,028 \\
\hline & PA s/ necrose & 31 & 4,2 & 0,9 & 4 & 2,4 & 6,6 & \\
\hline & PA branda & 65 & 3,8 & 0,6 & 3,8 & 2,5 & 5,4 & \\
\hline \multirow[t]{3}{*}{$\mathrm{Ht}$} & $\mathrm{PA} \mathrm{c} /$ necrose & 28 & 40,3 & 9,8 & 42 & 20 & 56 & 0,055 \\
\hline & PA s/ necrose & 30 & 36,8 & 8,8 & 37 & 20 & 56 & \\
\hline & PA branda & 70 & 40,7 & 7,2 & 41,5 & 13 & 54 & \\
\hline
\end{tabular}

$68,9 \%$ ), e para dosagem de creatinina sérica maior que $1 \mathrm{mg} / \mathrm{dl}$ (sensibilidade de 70,9\% e especificidade de 70,1\%).

Quanto a análise da etiologia observou-se que esta difere significativamente entre os grupos. A proporção de etiologia biliar foi significativamente maior no grupo com PA branda $(52,2 \%)$ do que nos grupos de PA grave com necrose $(34,3 \%)$ e sem necrose $(35,9 \%)$, com $p$ igual a 0,008 . Em contrapartida a proporção de "outras" etiologias no grupo com PA branda foi significativamente menor que nos grupos de PA grave com necrose $(42,9 \%)$ e sem necrose $(43,6 \%)$.

\section{DISCUSSÃO}

Até o momento não há método definitivo na avaliação da gravidade da PA, além do questionamento da real importância deste conhecimento e do seu impacto na evolução do paciente ${ }^{4}$.

Atualmente um sistema de avaliação de gravidade da PA bem aceito é o APACHE II ${ }^{5}$.

O fato é que os sistemas de avaliação incluem parâmetros clínicos e laboratoriais nem sempre disponíveis no prontuário, podendo levar até quarenta e oito horas para serem obtidos dados concretos sobre a gravidade do episódio da PA. De forma que ao obter-se o resultado destas avaliações, o paciente já pode apresentar sinais clínicos óbvios de deterioração de quadro ${ }^{6}$.

A estratificação do quadro clínico quanto à possibilidade do paciente evoluir com a forma grave da doença, visa antever as complicações clínicas, principalmente as relaciona- das às falências orgânicas, com a indicação de internação precoce em Centro de Tratamento Intensivo (CTI) e monitorização do paciente e tratamento precoce das intercorrências ${ }^{5}$. Apesar da redução da taxas de mortalidade com essas medidas, ainda não conseguimos interferir com os eventos fisiopatológicos em seu momento inicial. Na verdade, ao acreditar-se, por exemplo, que a hipocalcemia seria um critério de mau prognóstico segundo Ranson ${ }^{2}$, o paciente já está grave e "inflamado". Já houve alteração da permeabilidade capilar e conseqüentemente a albumina já se encontra no interstício junto com o cálcio ligado à mesma ${ }^{6}$. Além do que, por mais precoce que tenha sido a internação em CTI, só inicia-se o tratamento de uma complicação orgânica quando esta já se encontra instalada.

Estas limitações descritas geram uma discussão em torno do real papel dos escores prognósticos. A taxa de mortalidade realmente diminuiu pela utilização destes, ou porque paralelamente também houve melhorias em relação a assistência em CTIs, com melhor conhecimento clínico das complicações associado a utilização de novos recursos tecnológicos? ${ }^{8}$.

Em função disto a procura por um método simples e rápido tem sido constante, e investimentos científicos têm sido direcionados para testes bioquímicos que no entanto mostram resultados ainda abaixo da expectativa. A dosagem do PCR ainda tem sido aceita como padrão para avaliar a gravidade da PA, mas é uma proteína de fase aguda e inespecífica ${ }^{9}$, 10,5. Atenção tem sido dada a dosagem de TAPs (em inglês: "Trypsinogen Activation Peptide") que tem se mostrado sen- 
sível e específico além de poder ser dosado na urina, porém mais testes são necessários para estabelecer o seu papel na avaliação da $\mathrm{PA}^{9,11,5}$.

Não há intenção em desprestigiar nenhum método clínico-laboratorial atualmente utilizado para estratificar a PA, no entanto, enquanto não for descoberto um método rápido, simples, e acessível a todos os hospitais, ao mesmo tempo em que se obtenha um prognóstico da doença, estaremos tratando as complicações apenas após seu aparecimento e sem interferir com a fisiopatologia da PA em seus momentos iniciais.

Portanto para uma mudança significativa no curso da doença, esforços devem ser direcionados no sentido de conseguir tal método para avaliar o prognóstico, associado a um tratamento específico da PA que possa, por exemplo, interferir na evolução da cascata de reação inflamatória ou na ativação das enzimas no interior da glândula ${ }^{12,13}$.

A TC de abdômen tem importante papel no diagnóstico da necrose pancreática, porém o seu uso rotineiro na abordagem inicial do paciente com PA sem sinais clínicos de gravidade é discutível, inclusive tendo sido questionado um possível efeito negativo na evolução do episódio da PA e apresentando maiores taxas de necrose e infecção devido a efeitos do meio de contraste na microcirculação pancreática favorecendo a isquemia local ${ }^{14}$.

Diante deste contexto é possível perceber que o obtido é o diagnóstico precoce da pancreatite grave, e não o prognóstico. Ainda não temos como saber se o paciente vai evoluir mal ou não logo após o início do episódio de PA e antes do destino deste episódio, brando ou grave, estar aparentemente selado.

Então, já que as informações são limitadas, por quê não enfatizarmos esta avaliação inicial a partir de critérios mais simples? Critérios estes que poderiam ser baseados em informações e dados laboratoriais comumente presentes nos prontuários de pacientes que são admitidos com dor abdominal ou pancreatite, valorizando a avaliação clínica continuada.

Esta modalidade de avaliação já foi proposta por outros autores, identificando a dosagem de glicemia com sensibilidade de $79 \%$ a $81 \%$ e especificidade de $63 \%$ a $79 \%{ }^{15,6}$.

Fica claro que não existe teste bioquímico específico para o diagnóstico e prognóstico da PA. E ainda que os escores prognósticos por mais complexos que sejam apresentam sensibilidade e especificidade limitadas. O APACHE II chega a apresentar na admissão (a partir de oito pontos) sensibilidade de $68 \%$ e especificidade de $67 \%$ para o diagnóstico de PA grave $^{9}$. E sensibilidade de $36 \%$ e especificidade de $72 \%$ para diagnóstico de necrose pancreática ${ }^{16}$. Os critérios de Ranson (a partir de 3 pontos) apresenta sensibilidade de $75 \%$ e especificidade de $68 \%$, porém apenas após 48 horas $^{9,2}$.

$\mathrm{Na}$ verdade quando se fala em prognóstico de um evento de PA, a presença de necrose é o principal determinante da mortalidade, o que é agravado quando há complicações infecciosas locais. ${ }^{17-21}$.

A questão pertinente é como identificar cedo quais os pacientes que poderiam evoluir com a forma grave usandose critérios clínico-laboratoriais simples, e implementar precocemente medidas terapêuticas na tentativa de reduzir a morbimortalidade?
No total de pacientes estudados notou-se nitidamente a presença de um grupo que evoluiu de forma grave com taxa de mortalidade superior a da PA branda e que também foi inferior a taxa de mortalidade na PA grave com necrose. Neste grupo, à semelhança de outros autores ${ }^{18,22,23}$, o principal determinante da gravidade foi a falência orgânica sem necrose. Já que teoricamente a necrose não estava presente, o motivo para isto pode ser o comprometimento prévio de sistemas orgânicos que teria sido agravado pela PA. Por estas razões acreditamos que um outro estudo se torna necessário para a confirmação das doenças associadas nesse grupo e sua relação com episódio de PA no impacto sobre o paciente.

Não houve diferença entre os grupos estudados quanto a influência da idade na evolução do episódio de PA, ao contrário do proposto por Ranson ${ }^{2}$.

O tempo de internação foi significativamente maior nos pacientes com necrose pancreática (média de 35,7 dias), o que já era esperado pela maior morbidade em função da presença desta.

Quanto a aplicação do APACHE II para a avaliação dos nossos pacientes, este nunca estava presente na admissão. Tendo como maior limitação a ausência de dados de exames laboratoriais, dados referentes a Escala de Coma de Glasgow (ECG) e relatos sobre a presença de doenças associadas, todos necessários para o cálculo.

O fator tempo de evolução e a ausência de dados laboratoriais são os principais fatores limitantes na classificação do episódio de PA em nosso hospital, e infelizmente em muitos outros. Desta forma, quando propomos a avaliação clínicolaboratorial inicial simplificada, um dos objetivos é adequar a avaliação do paciente à nossa realidade social e econômica, além de torná-la prática detectando fatores de risco para a evolução desfavorável do paciente na primeira avaliação independente do tempo de evolução e da necessidade de exames laboratoriais com ensaios mais complexos e de custo elevado ${ }^{5,13,15}$.

Dos exames avaliados foi observado que o grau de elevação da amilase não mostra relação com a gravidade, como já descrito por outros autores e exposto anteriormente ${ }^{9,24}$.

Ao contrário dos critérios estabelecidos por Ranson ${ }^{2}$ a leucocitose não mostrou relação com a gravidade ou presença de necrose nos casos estudados.

A avaliação do hematócrito também não mostrou variação importante entre os grupos estudados, não havendo relação entre esta medida e a gravidade da doença, como proposto por outros autores ${ }^{5,25}$.

Um dado consistente são os níveis elevados de glicemia nos pacientes com necrose (média de $210,5 \mathrm{mg} / \mathrm{dl}$ ) significativamente maior do que nos pacientes com PA branda ou PA grave sem necrose. Tal fato pode estar associado a preservação anatômica e funcional do pâncreas na ausência de necrose mantendo a produção de insulina com conseqüente controle da glicemia, visto que os níveis glicêmicos na ausência de necrose foram próximos aos normais $(140,08 \mathrm{mg} / \mathrm{dl} \mathrm{e}$ $140,30 \mathrm{mg} / \mathrm{dl}$ respectivamente na PA branda e PA grave sem necrose). Portanto a elevação da glicemia pode ser um dado indireto da presença de necrose pancreática. Inclusive outros autores já propuseram a glicemia como uma forma de avaliação simplificada ${ }^{6,15}$. 
As dosagens de uréia e creatinina apresentaram-se significativamente menores nos pacientes com PA branda, mantendo-se dentro da normalidade, não havendo diferença entre os grupos com PA grave com e sem necrose onde as medidas apresentavam-se anormalmente elevadas. Destas, a dosagem de creatinina é mais fidedigna por melhor refletir o nível de disfunção renal, além de possuir o $p$ valor de 0,0001 contra o $p$ valor da uréia de 0,0005 . Estes achados já foram reproduzidos por outros autores ${ }^{15,6,13}$.

Para a dosagem de potássio houve diferença estatística significativa a favor do grupo com PA grave sem necrose, onde o nível médio foi maior que o grupo com PA branda e com PA grave com necrose. Curiosamente, em todos os grupos os valores médios mantiveram-se na faixa da normalidade, de maneira que a diferença estatística não implica em importância clínica.

Dentre todos os dados de exames físicos pesquisados na admissão o único que apresentou diferença significativa entre os grupos foi a taquicardia, apresentando-se em proporção inferior no grupo com PA branda quando comparado com os grupos com PA grave com e sem necrose, onde esta se encontrava em maior proporção e sem diferença significativa entre ambas.

Finalmente observou-se que a proporção de óbitos difere significativamente entre os três grupos. Como a proporção de pacientes com PA grave sem necrose mostrou-se elevada, questionou-se se estes pacientes não poderiam ter sido classificados erroneamente como sem necrose, de fato com a elevada taxa de TC sem contraste seria de se esperar que em certos casos a necrose não fosse diagnosticada. No entanto este grupo apresenta características de mortalidade própria e superior aos da PA branda. No entanto casos de PA grave associados à falência orgânica múltipla, na ausência de necrose, têm sido relatados $6,18,22,23$.

Mesmo no grupo com PA grave com necrose a mortalidade foi muito elevada $(65,7 \%)$, quando comparadas com outros centros onde esta pode variar entre 14 e 42\% $\%, 6,13$. Este fato pode estar associado a baixa taxa de internação em CTI $(18,3 \%)$ o que implicou em tratamento inadequado a uma parcela dos pacientes, ou ao momento escolhido para a intervenção cirúrgica ${ }^{26}$.

Observa-se então que uma população estudada terá seu perfil variando em função do grau de complexidade da unidade hospitalar avaliada, e também de características regionais presentes. De modo que qualquer sistema de classificação aplicado deve levar em consideração tais particularidades, e que sistemas rígidos não necessariamente atenderão à todas as populações.

Nessa população observou-se que níveis séricos acima da normalidade de glicose, uréia e creatinina são isoladamente associados, de modo significativo, às complicações nos episódios de PA e conseqüentemente à maior mortalidade.

A presença de apenas um deles, em particular a glicemia elevada, reforça a necessidade da realização de TC de abdômen para a detecção de possível necrose, e instalação de medidas diagnósticas e terapêuticas cabíveis, visando reduzir a morbidade e mortalidade.

O ponto de corte que expressa a melhor relação entre a sensibilidade e especificidade do nível de glicemia em relação a presença de necrose é o de maior que $121 \mathrm{mg} / \mathrm{dl}$, com sensibilidade de $63,3 \%$ e especificidade de $61,2 \%$ para esta população, que são próximas ao do APACHE II na admissão para gravidade, e melhor que este na detecção de necrose, porém ainda aquém do desejado.

Os pontos de corte para dosagem de uréia e creatinina, apesar de detectados, não oferecem aplicabilidade prática pois encontram-se dentro da faixa de normalidade.

Mais importante que a presença de taquicardia nos pacientes graves, é a sua ausência nos casos de PA branda, que praticamente afasta a possibilidade de uma doença grave em andamento em função de sua especificidade de $92 \%$. Por outro lado apresenta baixa sensibilidade (29\%), o que a torna pouco útil como critério de gravidade.

De acordo com os resultados deste estudo o principal determinante da mortalidade é a presença de necrose pancreática, porém a presença de coleção pancreática e insuficiência orgânica sem necrose também apresentaram mortalidade significativa. Observamos que na admissão do paciente a ausência de taquicardia estava associada à forma branda da doença, enquanto que os níveis plasmáticos de uréia e creatinina elevados estavam associados à forma grave da doença e a hiperglicemia à necrose pancreática.

Inferimos, desta forma, que a avaliação inicial simplificada ainda tem espaço, embora limitado, na avaliação prognóstica do paciente com PA.

\begin{abstract}
Background: Our goal was to study if early and simplified clinical and laboratorial evaluation are associated to severity and necrosis in acute panceatitis (AP). Method: One hundred and sixty four patients, hospitalized at the Clementino Fraga Filho University Hospital between January 1990 and August 2002 with a AP final diagnosis, were included in this study. The study was retrospective and included only the first episode of each patient. From the charts we obtained clinical, laboratorial, imaging exams, operations and death data. From these data we established the severity of each case. Statistical analysis used the Variance Analysis test of Kruskal-Wallis, completed by the Multiple Comparisons test if the diference was significant. Statistic significance was defined as $p<0,05$. Results: Biliar etiology was the most common: 43,9\% of cases, necrosis incidence was 21,3\% and overall mortality was $23,2 \%$. We observed that normal cardiac frequency at admission was associated to mild AP, as high urea and creatinine plasmatic levels at admission to severe AP, and hyperglycemia $(>121 \mathrm{mg} / \mathrm{dl})$ to pancreatic necrosis. Conclusions: We concluded that early and simplified clinical and laboratorial evaluation at admission had a limited but clear role in AP prognosis.
\end{abstract}

Key words: Pancreatitis; Acute disease; Pancreatitis, acute necrotizing; Prognosis. 


\section{REFERÊNCIAS}

1. Bradley EL $3^{\text {rd }}$. The necessity for a clinical classification of acute pancreatitis the Atlanta system. In: Bradley EL $3^{\text {rd }}$. Acute pancreatitis diagnosis and therapy. New York: Raven Press; 1994. p. 27-32.

2. Ranson JH, Rifkind KM, Roses DF, Fink SD, Eng K, Localio SA. Objective early identification of severe acute pancreatitis. Am J Gastroenterol. 1974;61(6):443-51.

3. Imrie CW, Whyte AS. A prospective study of acute pancreatitis. Br J Surg. 1975; 62(6):490-4.

4. McKay CJ, Imrie CW. Staging of acute pancreatitis. Is it important? Surg Clin North Am. 1999;79(4):733-43.

5. Dervenis CG. Staging acute pancreatitis: where are we now? Pancreatology. 2001;1(3):201-6.

6. Blum T, Maisonneuve P, Lowenfels AB, Lankisch PG. Fatal outcome in acute pancreatitis: its occurrence and early prediction. Pancreatology. 2001;1(3):237-41.

7. Smotkin J, Tenner S. Laboratory diagnostic tests in acute pancreatitis. J Clin Gastroenterol. 2002;34(4):459-62.

8. Bank S, Singh P, Pooran N, Stark B. Evaluation of factors that have reduced mortality from acute pancreatitis over the past 20 years. J Clin Gastroenterol. 2002;35(1):50-60.

9. Triester SL, Kowdley KV. Prognostic factors in acute pancreatitis. J Clin Gastroenterol. 2002;34(2):167-76.

10. Büchler M, Uhl W, Malfertheiner P. Biochemical staging of acute pancreatitis. In: Beger HG, Büchler M, editors. Acute pancreatitis. Berlin: Springer-Verlag; 1987. p. 143-53.

11. Kylanpaa-Back M, Kemppainen E, Puolakkainen P, Hedstrom J, Haapiainen R, Perhoniemi V, Kivilaakso E, Korvuo A, Stenman U. Reliable screening for acute pancreatitis with rapid urine trypsinogen-2 test strip. Br J Surg. 2000;87(1):49-52.

12. Uhl W, Schrag HJ, Schmitter N, Aufenanger J, Nevalainen TJ, Buchler MW. Experimental study of novel phospholipase $\mathrm{A}_{2}$ inhibitors in acute pancreatitis. Br J Surg. 1998;85(5):618-23.

13. Halonen KI, Leppaniemi AK, Lundin JE, , Kemppainen EA, Haapiainen RK. Predicting fatal outcome in the early phase of severe acute pancreatitis by using novel prognostic models. Pancreatology. 2003;3(4):309-15.

14. Carmona-Sanchez R, Uscanga L, Bezaury-Rivas P, RoblesDiaz G, Suazo-Barahona J, Vargas-Vorackova F. Potential harmful effect of iodinated intravenous contrast medium on the clinical course of mild acute pancreatitis. Arch Surg. 2000;135(11):1280-84.

15. Meek K, Toosie K, Stabile BE, Elbassir M, Murrell Z, Lewis RJ, Chang L, de Virgilio C. Simplified admission criterion for predicting severe complications of gallstone pancreatitis. Arch Surg. 2000;135(9):1048-52; discussion 1052-4.
16. Lankisch PG, Warnecke B, Bruns D, Werner HM, Grossmann F, Struckmann K, Brinkmann G, Maisonneuve P, Lowenfels AB. The APACHE II score is unreliable to diagnose necrotizing pancreatitis on admission to hospital. Pancreas. 2002; 24(3):217-22.

17. Bittner R. Pancreatic abscess and injected pancreatic necrosis: different local septic complications in acute pancreatitis. In: Beger HG, Büchler M, editors. Acute pancreatitis. Berlin: SpringerVerlag; 1987. p. 216-23.

18. Buchler MW, Gloor B, Muller CA, Friess H, Seiler CA, Uhl W. Acute necrotizing pancreatitis: treatment strategy according to the status of infection. Ann Surg. 2000;232(5):619-26.

19. Beger HG, Kunz R, Bittner R. Prognostic criteria in necrotizing pancreatitis. In: Beger HG, Büchler M, editors. Acute pancreatitis. Berlin: Springer Verlag; 1987. p.198-200.

20. Beger HG. Surgery in acute pancreatitis. Hepatogastroenterology. 1991;38(2):92-6.

21. Büchler P, Reber HA. Surgical aproach in patients with acute pancreatitis. Is infected or sterile necrosis an indication? In whom, should this be done, when and why? Gastroenterol Clin North Am. 1991;28(3):661-71.

22. Buter A, Imrie CW, Carter CR, Evans S, McKay CJ. Dynamic nature of early organ dysfunction determines outcome in acute pancreatitis. Br J Surg. 2002; 89(3):298-302.

23. De Beaux AC, Palmer KR, Carter DC. Factors influencing morbidity and mortality in acute Pancreatitis: an analysis of 279 cases. Gut. 1995;37(1):121-6.

24. Yadav D, Agarwal N, Pitchumoni CS. A critical evaluation of laboratory tests in acute pancreatitis. Am J Gastroenterol. 2002;97(6):1309-18.

25. Lankisch PG, Mahlke R, Blum T, Bruns A, Bruns D, Maisonneuve P, Lowenfels AB. Hemoconcentration: an early marker of severe and or necrotizing pancreatitis? A critical appraisal. Am J Gastroenterol. 2001;96(7):2081-5.

26. Renteria JM. Pancreatite aguda necrosante: tratamento operatório [Dissertação]. Rio de Janeiro (RJ): Universidade Federal do Rio de Janeiro; 1999.

Como citar este artigo:

Carneiro MC, Manso JEF, Eulálio JMR, Renteria JM. O papel da avaliação inicial simplificada no prognóstico da pancreatite aguda. Rev Col Bras Cir. [periódico na Internet] 2006 Mai-Jun; 33(3). Disponível em URL: http://scielo.br/rcbc

Endereço para correspondência:

Márcio Cavalcante Carneiro

Av. Guilherme de Almeida, 633 / apto. 204

Recreio dos Bandeirantes

CEP: 22790-100 - Rio de Janeiro - RJ

E-mail: carneiromc@globo.com 